\title{
Digital Image Analysis of Internal Light Spots of Appressoria of Colletotrichum acutatum
}

\author{
J. Diéguez-Uribeondo, H. Förster, and J. E. Adaskaveg
}

Department of Plant Pathology, University of California, Riverside 92521.

Accepted for publication 2 February 2003.

\begin{abstract}
Diéguez-Uribeondo, J., Förster, H., and Adaskaveg, J. E. 2003. Digital image analysis of internal light spots of appressoria of Colletotrichum acutatum. Phytopathology 93:923-930.

The initial penetration process of appressoria of Colletotrichum acutatum on almond leaves was studied using digital image analysis of light micrographs and scanning electron microscopy. For image analysis, a series of sequential, partially focused digital micrographs of appressoria was analyzed to generate a single, completely focused montage image with a continuous in-focus depth of field. In studies on the development of the internal light spot (ILS), we observed that $50.4 \%$ of the appressoria formed an ILS after leaves were inoculated and incubated for $12 \mathrm{~h}$ at

and line profile software options showed that the ILS had a depth relief that was below that of the leaf surface. Depth relief analysis in the ILS region during incubation revealed an increase in depth in this area of up to $1.8 \mu \mathrm{m}$ in some of the appressoria. A comparative morphological study of the ILS in montage images and the penetration pore of appressoria in scanning electron micrographs showed similar shapes and dimensions of the two structures in the appressorium. Light micrographs of histological sections confirmed fungal penetration and internal vesicle formation in almond leaves within $36 \mathrm{~h}$ after inoculation and incubation at $20^{\circ} \mathrm{C}$. This study represents the first direct evidence that the ILS in appressoria corresponds to the penetration pore and the developing penetration peg using a rapid, digital image analysis technique.
\end{abstract} $20^{\circ} \mathrm{C}$, and that this increased to $95.8 \%$ after $24 \mathrm{~h}$. Comparative image analyses of appressoria with and without ILSs using depth relief mapping
Additional keywords: almond, histology, quiescent infection.
The penetration process is a critical stage in any plant-pathogen interaction (14). Understanding this process and knowing conditions that are favorable for disease is important to better comprehend the manifestation of fungal diseases. Host infection has been extensively studied in Colletotrichum spp. (24). The genus Colletotrichum comprises plant pathogens that cause economically important diseases such as anthracnoses and blights on many crops worldwide (28). In California, almond anthracnose caused by $C$. acutatum J. H. Simmonds can be a serious disease resulting in substantial economic losses $(1,2,8)$. In the genus Colletotrichum, the establishment of an infection usually relies on a sequence of early penetration events that occur in specialized infection structures, the appressoria (21). The appressoria of Colletotrichum spp. are globose, melanized cells from which infection pegs emerge and directly penetrate the host cell wall. Among Colletotrichum spp. and other ascomycetous pathogenic fungi, appressorial development appears to be very similar (14). The hyphal apex expands and the appressorium matures and becomes melanized. For host infection, a penetration pore and peg are formed at the base of the appressorium $(3,19,27)$. The appressorium becomes an asymmetric polarized cell, with an upper dome region and a basal region containing the pore apparatus $(22,24)$. In some species, the penetration pore is surrounded by a new wall overlay $(25,29)$. Alternatively, a new wall structure, the appressorial cone $(5,15)$, forms around the pore and gives rise to the penetration peg (20).

During appressorial development in Colletotrichum spp., an internal light spot (ILS) becomes visible within the appressorium using brightfield microscopy (23). The presence of the ILS has generally been associated with the formation of the penetration

Corresponding author: J. E. Adaskaveg; E-mail address: jim.adaskaveg@ucr.edu

Publication no. P-2003-0522-01R

(c) 2003 The American Phytopathological Society pore and host penetration. Thus, Zulfiqar et al. (32), in a study on the infection of citrus tissues by $C$. acutatum and C. gloeosporioides, associated the presence of ILSs in appressoria with the penetration peg and the subsequent formation of quiescent infections. In other studies on early penetration and host colonization events by $C$. acutatum, however, different interpretations have been presented. For example, Leandro et al. (18) reported that although small, basal, hyaline openings at the center of the appressoria (the ILSs by our definition) were identified, no infection pegs were seen and no quiescent infections developed in symptomless strawberry leaves inoculated with $C$. acutatum. No cytological investigations have been done previously on $C$. acutatum infecting almond (Prunus dulcis (Mill.) D. A. Webb) leaves. In addition, no direct correlation of the ILS with an infection structure has been made in any of the studies mentioned above. Experiments to elucidate the nature of the ILS and its association with the penetration process are not simple because time-consuming and expensive techniques can be involved. In light and electron microscopy studies, resin-embedded plant and fungal cell walls tend to separate at the junction between the two walls during sectioning, making it difficult to obtain good median sections of the thin penetrating hypha $(11,12)$. Thus, quantification of specific events in the fungal penetration process can be a very time consuming task; consequently, more simple and rapid techniques need to be developed to allow analysis of a large sample size.

Recently, new computer software programs for digital image analysis have been introduced that allow the study of samples with a large depth of field at the microscopic level. These programs combine the in-focus regions from a series of sequential images taken of a sample, each in a different plane of focus, to generate a single in-focus image. Three-dimensional models can be generated to obtain a quantitative image interpretation. In our research, we used this technology to study appressoria of $C$. $a c u$ tatum during the penetration of almond leaves and to elucidate the nature of the ILS. 


\section{MATERIALS AND METHODS}

Inoculum preparation and inoculation of almond leaves. An isolate of the pink phenotype of $C$. acutatum, the most predominant strain in California orchards (8), was used in experiments. Conidial suspensions $\left(10^{6}\right.$ conidia/ml $)$ in sterile distilled water were prepared from 14-day-old potato dextrose agar cultures grown at $23 \pm 2{ }^{\circ} \mathrm{C}$ in the laboratory. Potted almond plants (cv. Carmel) were grown in the greenhouse. During experiments, they were maintained under controlled conditions in growth chambers at $20 \pm 2^{\circ} \mathrm{C}$ with a 12 -h light $(12,000$ to 15,000 lux, Sylvania Cool White F72T12/CW/VHO) and 12-h dark cycle. Attached leaves were inoculated with $10-\mu l$ droplets of the conidial suspension, with droplets spaced $5 \mathrm{~mm}$ apart on the lower leaf surface. Parafilm-sealed petri dishes supported by stakes were used as incubation chambers to maintain a relative humidity of $>95 \%$. Leaves were incubated for $0,3,6,12,24$, and $48 \mathrm{~h}$ after inoculation. Each incubation time was replicated on five different plants and the experiment was done twice.

Appressoria formation and ILS development. To evaluate appressorium formation and development of the ILS, five tissue samples approximately 2 by $2 \mathrm{~mm}$ in size were excised from drop-

TABLE 1. Time sequence of appressorial development of Colletotrichum acutatum on almond leaves

\begin{tabular}{lccc}
\hline $\begin{array}{l}\text { Wetness duration } \\
\text { after inoculation }(\mathrm{h})^{\mathrm{w}} \mathrm{w}\end{array}$ & $\begin{array}{c}\text { Germ tubes with } \\
\text { appressoria }(\%)^{\mathrm{x}}\end{array}$ & $\begin{array}{c}\text { Melanized } \\
\text { appressoria }(\%)^{\mathrm{y}}\end{array}$ & $\begin{array}{c}\text { Appressoria with } \\
\text { ILSs }(\%)^{\mathrm{z}}\end{array}$ \\
\hline 3 & $0.0 \pm 0.0$ & $0.0 \pm 0.0$ & $0.0 \pm 0.0$ \\
6 & $2.3 \pm 1.5$ & $4.9 \pm 4.4$ & $0.0 \pm 0.0$ \\
12 & $90.1 \pm 3.8$ & $95.5 \pm 2.1$ & $51.3 \pm 4.6$ \\
24 & $99.2 \pm 0.8$ & $98.6 \pm 2.3$ & $92.4 \pm 2.1$ \\
48 & $100.0 \pm 0.0$ & $100.0 \pm 0.0$ & $100.0 \pm 0.0$ \\
\hline
\end{tabular}

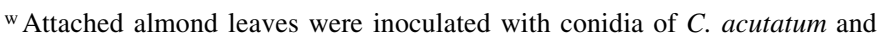
provided with selected wetness periods. Leaf samples were cleared in chloral hydrate and examined using light microscopy. A minimum of 300 conidia were examined for each category described below.

${ }^{x}$ Average incidence $(\%) \pm$ standard error was based on (number of germ tubes with appressoria/total number of germinated conidia) $\times 100$.

y Average incidence (\%) \pm standard error was based on (number of melanized appressoria/total number of appressoria) $\times 100$.

${ }^{z}$ Average incidence $(\%) \pm$ standard error was based on (number of melanized appressoria with an internal light spots [ILSs]/total number of appressoria) $\times 100$.

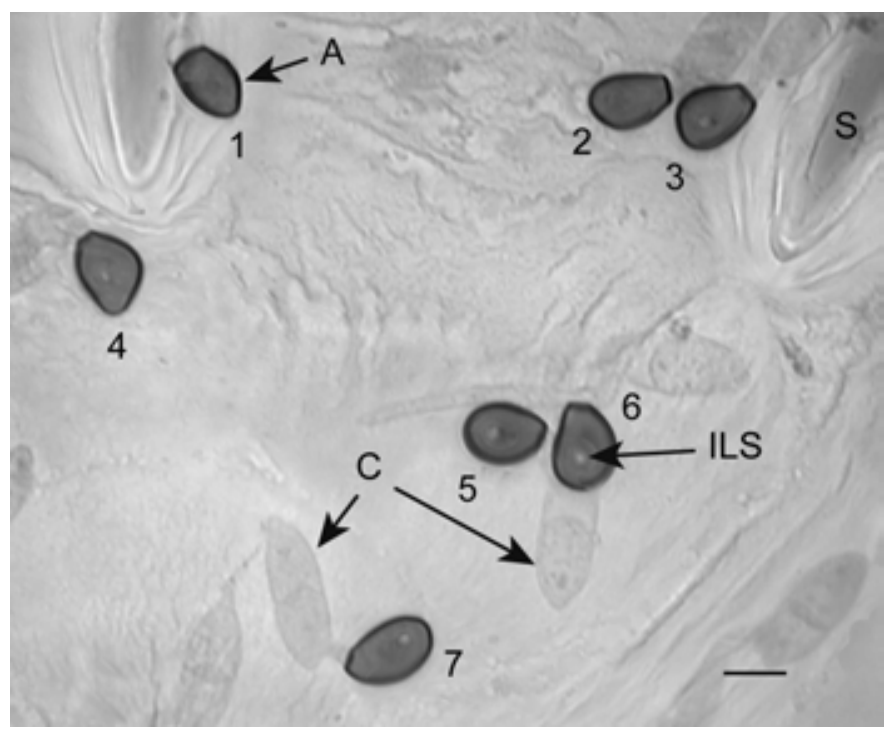

Fig. 1. Montage image of seven appressoria (A) of Colletotrichum acutatum on an almond leaf $12 \mathrm{~h}$ after inoculation. Two types of appressoria can be distinguished: appressoria without an internal light spot (ILS) (appressoria 1 and 2) and appressoria with an ILS (appressoria 3 to 7). $\mathrm{Bar}=5 \mu \mathrm{m} ; \mathrm{C}=$ conidium, $\mathrm{S}=$ stoma. let-inoculated leaves. Samples were cleared in saturated chloral hydrate for at least 2 days. For observation, samples were stained with $0.05 \%$ acid fuchsin for $2 \mathrm{~min}$, washed in distilled water, and mounted in water on glass slides. Samples were observed using brightfield microscopy (Zeiss Axioskop, Zeiss, Germany) at a magnification of $\times 400$ (10 by 40 ) or $\times 1,000$ (10 by 100$)$. The numbers of conidia with appressoria, melanized appressoria, and appressoria with ILSs were assessed by counting at least 300 conidia per sample in randomly chosen objective fields. The percentage of appressoria was calculated based on the total number of germinated conidia. The percentage of melanized appressoria was assessed as the number of dark-brown, rounded appressoria of the total number of appressoria. The percentage of appressoria with ILSs was calculated as the number of melanized appressoria showing a clear white halo of the total appressoria. The experiment was done three times.

Image capture and digital image analysis of light micrographs. Images from 20 appressoria at each postinoculation period were analyzed. Appressoria were photographed using a digital camera (Sony DKC-5000; Sony Corp. of America, Montvale, NJ) mounted on a Zeiss Axioskop compound microscope. Sequential images of appressoria 12, 24, and $48 \mathrm{~h}$ after inoculation at step-wise focus intervals of $\approx 1 \mu \mathrm{m}$ were captured using image capture software and the program Syncroscopy Auto-Montage (Microbiology International Inc., Frederick, MD). Approximately six images were used to generate a single in-focus (montage) image with the program options "blended depth" and a "patch size" between 5 and 20 pixels. Depth relief and line profile studies for the three-dimensional analysis were done on appressoria and ILS regions. Appressoria were selected that were not located above adjoining plant cell walls. To analyze depth relief in appressoria without ILSs, two perpendicular line profiles 10 to $15 \mu \mathrm{m}$ long crossing the center of the appressorium were studied. For appressoria with ILSs, the line profiles crossed the ILS. Similarly, two perpendicular line profiles were analyzed for a more detailed study of the ILS region.

Carbon particles, prepared as described by Grove et al. (10) and Bartnicki-Garcia et al. (4), were used to mark the leaf surface during these studies. The washed and centrifuged waterproof drawing ink (Black India Ink 4415; Eberhard Faber, Inc., Lewisburg, TN) particles were autoclaved for $20 \mathrm{~min}$ and stored at $4{ }^{\circ} \mathrm{C}$. Before use, $200 \mu \mathrm{l}$ of the autoclaved carbon particle suspension were centrifuged and the pellet was resuspended in four volumes of sterile distilled water. One or two drops of the suspension were added to leaf tissue samples that were cleared with chloral hydrate. A coverslip was placed on the sample and carbon particles were allowed to settle on the leaf surface for a half-hour before microscopy.

Digital image analysis of light micrographs and scanning electron microscopy of tape-mounted, inverted appressoria. Almond leaves were inoculated with a conidial suspension of $C$. acutatum as described above. After 12, 24, and $48 \mathrm{~h}$, inoculation droplets were carefully dried using kimwipes. Appressoria were then detached from the inoculated leaf area using transparent tape.

TABLE 2. Comparison of appressorial dimensions of Colletotrichum acutatum using digital image analysis of light micrographs (DIA-LM) and scanning electron microscopy $(\mathrm{SEM})^{\mathrm{z}}$

\begin{tabular}{lcc}
\hline Technique & ILS/pore diameter $(\mu \mathrm{m})$ & Ring diameter $(\mu \mathrm{m})$ \\
\hline DIA-LM & $0.8 \mathrm{a}$ & $1.4 \mathrm{a}$ \\
SEM & $0.7 \mathrm{a}$ & $1.4 \mathrm{a}$ \\
\hline
\end{tabular}

${ }_{\mathrm{z}}$ The internal light spot (ILS) was measured using DIA-LM, whereas pore diameter was measured using SEM. Mean values were determined from measurements of 30 appressoria for each technique. Values within each column followed by the same letter are not significantly different $(P>$ 0.05 ) based on an analysis of variance and least significant difference mean separation procedures. 

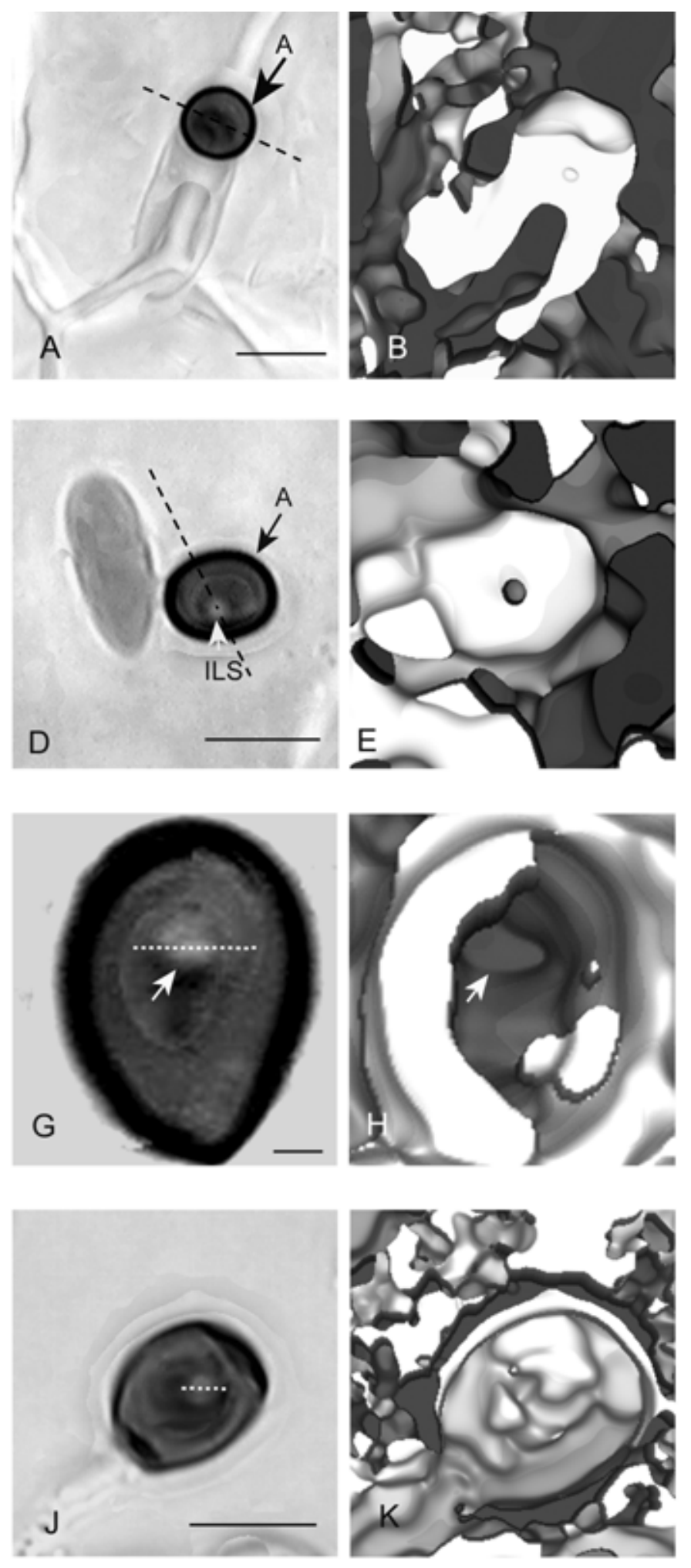
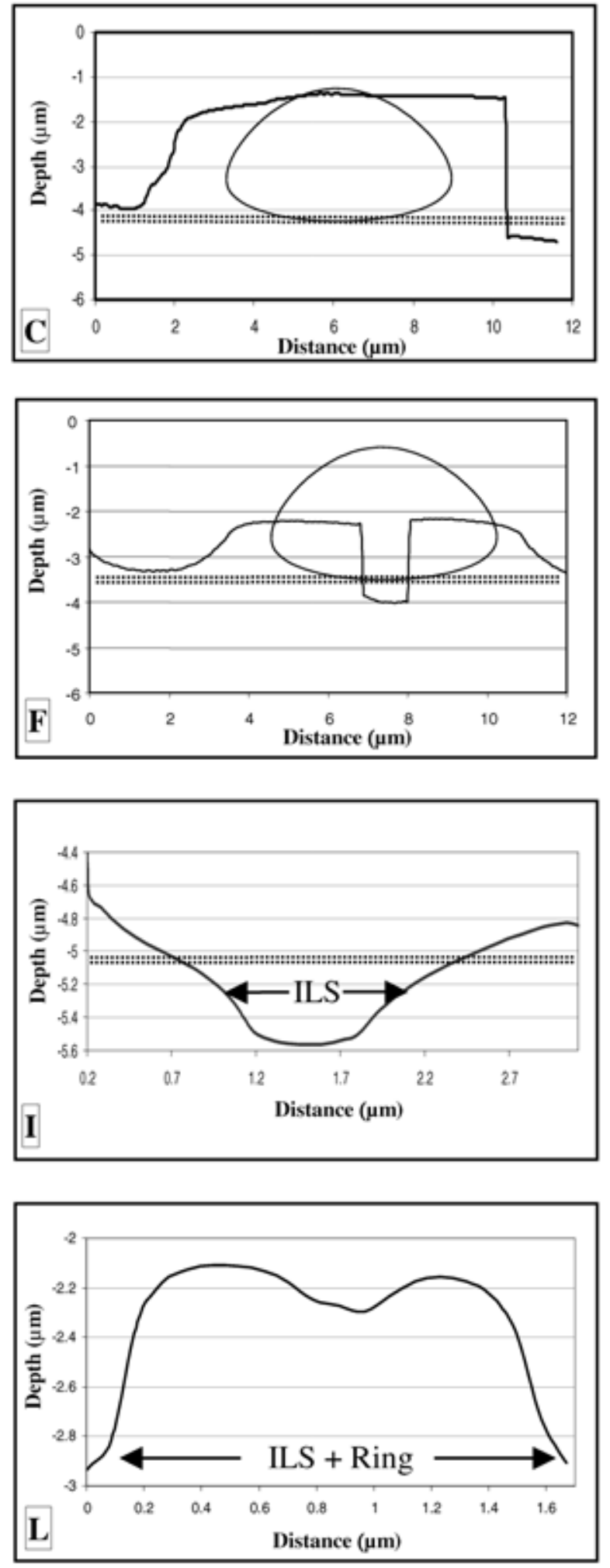

$-1 \mu \mathrm{m}$

$-6 \mu \mathrm{m}$

Fig. 2. Digital image analysis of appressoria (A) and internal light spots (ILSs) of Colletotrichum acutatum on almond leaves inoculated and incubated at $20^{\circ} \mathrm{C}$. $\mathbf{A}, \mathbf{D}, \mathbf{G}$, and $\mathbf{J}$ are montage images. The lines crossing the appressoria indicate where line profile analyses were done. B, E, $\mathbf{H ,}$ and $\mathbf{K}$ are depth relief maps. Relief is indicated by the gray scale bar that extends from $-1 \mu \mathrm{m}$ (light gray) to $-6 \mu \mathrm{m}$ (dark gray). $\mathbf{C}, \mathbf{F}, \mathbf{I}$, and $\mathbf{L}$ are line profiles. The double line in each line profile indicates the estimated leaf surface. A, Montage image of an appressorium with no ILS at $12 \mathrm{~h}$ after inoculation. Bar $=5 \mu \mathrm{m}$. B, The appressorium has no depth relief below the leaf surface. C, The line profile shows that depth relief in the appressorium is above the estimated leaf surface. D, Appressorium with ILS. Bar $=5 \mu \mathrm{m}$. E, The appressorium has depth relief. F, The line profile indicates that its lowest depth is below that of the leaf surface. G, Higher magnification of an appressorium with ILS $24 \mathrm{~h}$ after inoculation. The ILS is surrounded by a dark ring (arrow). Bar $=1 \mu \mathrm{m}$. H, Depth relief and I, line profile indicate that depth relief is present in the ILS region that extends below the leaf surface. J, Inverted tape-mounted appressorium $24 \mathrm{~h}$ after inoculation. $\mathbf{K}$, Depth relief-map showing a structure protruding out of the ILS region. $\mathbf{L}$, The line profile suggests that the protruding structure is the penetration peg. Bar $=5 \mu \mathrm{m}$. 

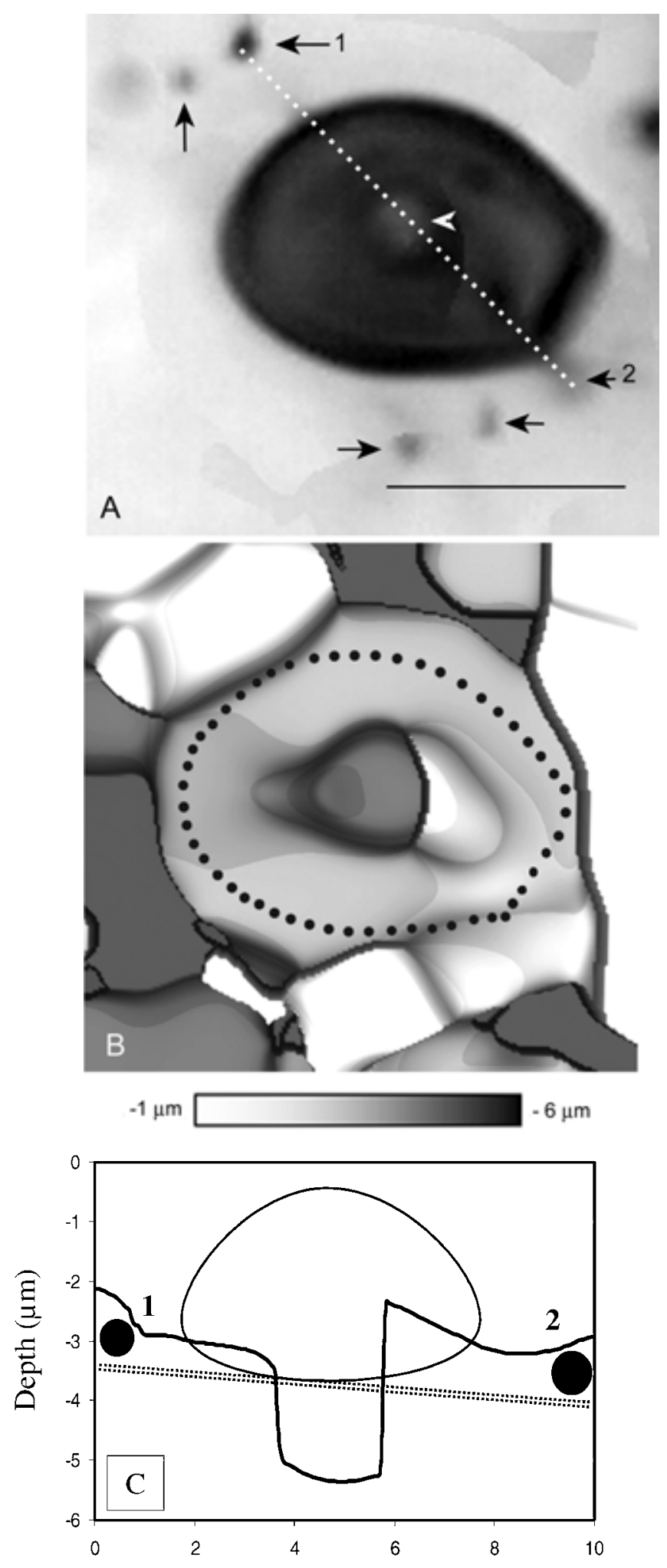

Distance $(\mu \mathrm{m})$

Fig. 3. Leaf surface calibration of digital images using carbon particles adjacent to an appressorium with an internal light spot (ILS) of Colletotrichum acutatum on an almond leaf inoculated and incubated for $48 \mathrm{~h}$ at $20^{\circ} \mathrm{C}$. A, Montage image of the appressorium with adjacent carbon particles (arrows). The arrowhead indicates the ring surrounding the ILS. The line crossing the appressorium from particle 1 to 2 is where line profile analysis was done. Bar $=5 \mu \mathrm{m}$. B, Relief map. Depth relief is indicated by the gray scale bar that extends from $-1 \mu \mathrm{m}$ (light gray) to $-6 \mu \mathrm{m}$ (dark gray). $\mathbf{C}$, Line profile between carbon particles 1 and 2 showing that the depth relief in the ILS is below the leaf surface that is indicated by the double line.
For digital-image analysis, the tape-mounted appressoria were examined using brightfield microscopy as described above.

For scanning electron microscopy (SEM), samples with tapemounted appressoria were fixed in $2.5 \%$ glutaraldehyde in $0.05 \mathrm{M}$ sodium phosphate buffer ( $\mathrm{pH}$ 6.8) for 18 to $24 \mathrm{~h}$. Samples were washed three times in phosphate buffer, postfixed in $2 \%$ osmium tetroxide for $2 \mathrm{~h}$ to improve specimen fixation (9), and rinsed twice in distilled water. Samples were dehydrated in a series of ethanol $(20,50,80,95$, and $100 \%)$ and chemically dried by using a series $(1: 2,1: 1,2: 1,1: 0$, and 1:0 dilution) of hexamethyldisilizane (Ted Pella, Inc., Redding, CA) in ethanol. Samples were mounted on SEM stubs, sputter coated with gold to a thickness of 15 to $20 \mathrm{~nm}$ using a Polaron ES5000 Magnotron Cool-head Coating System (Bio-Rad Laboratories, Richmond, CA), and observed using a scanning electron microscope (Model XL30-FEG; FEI Philips Inc., Hillsboro, OR). Thirty appressoria were morphologically characterized using SEM.

Histology of fungal penetration and colonization. For histological studies on fungal penetration and colonization, inoculated almond leaf samples ( 2 by $2 \mathrm{~mm}$ ) were fixed in $2.5 \%$ glutaraldehyde in $0.05 \mathrm{M}$ phosphate buffer ( $\mathrm{pH}$ 6.8) for 18 to $24 \mathrm{~h}$ at room temperature. Samples were rinsed three times for $15 \mathrm{~min}$ per wash in $0.05 \mathrm{M}$ phosphate buffer ( $\mathrm{pH}$ 6.8) and dehydrated in a series of ethanol $(10,35,50,70,80$, and $95 \%)$ three times for 5 min per concentration. Samples were embedded in JB-4 embedding medium (Polysciences, Inc., Warrington, PA) using a 1:2, 1:1, 2:1, 1:0, and 1:0 dilution of resin in ethanol. Transverse sections $\approx 3 \mu \mathrm{m}$ in thickness were obtained using a rotary microtome (Model 820; American Optical, Buffalo, NY). Sections were stained with $0.05 \%$ acid fuchsin for $2 \mathrm{~min}$, washed in distilled water, and mounted on glass slides for light microscopy. Thirty appressoria were morphologically characterized using this method.

Data analysis. Data on appressorial development (i.e., conidia with appressoria, melanized appressoria, and appressoria with ILSs) were summarized by calculating mean and standard error values for selected sampling times. The dimensions of the ILS and the appressorial pore using light and SEM, respectively, were evaluated using analysis of variance and least significant difference mean separation procedures. Regression analysis was performed for mean depth relief data of the ILS of appressoria at selected times after inoculation using the statistical analysis system or SAS (version 6.12; SAS Institute, Cary, NC).

\section{RESULTS}

Appressoria formation and ILS development. In a time-sequence study, conidia of $C$. acutatum started germinating on al-

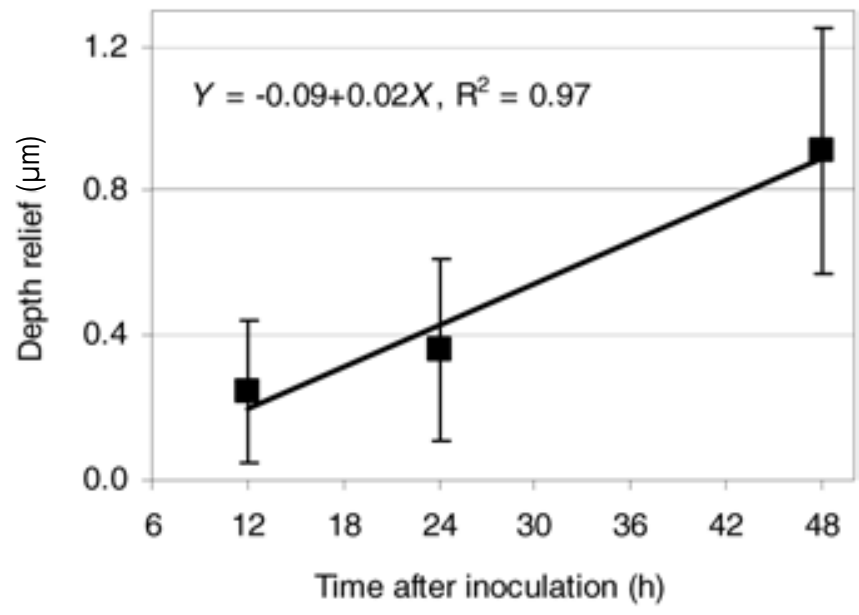

Fig. 4. Regression of depth relief in the internal light spots of appressoria of Colletotrichum acutatum on time after inoculation of almond leaves at $20^{\circ} \mathrm{C}$. 
mond leaf tissue $3 \mathrm{~h}$ after inoculation and incubation at $20^{\circ} \mathrm{C}$. Differentiation of the germ tube apex into appressoria began $6 \mathrm{~h}$ after inoculation (Table 1). By $12 \mathrm{~h}$ after inoculation, the majority of germ tubes $(90.1 \%)$ had differentiated into appressoria and $95.5 \%$ of the appressoria were melanized. At this time, an ILS became apparent in $51.3 \%$ of the appressoria and could be clearly observed using brightfield microscopy (Fig. 1). Thus, there were three types of appressoria, non-melanized appressoria, and melanized appressoria with and without an ILS. The proportion of appressoria with an ILS increased to $92.4 \%$ at $24 \mathrm{~h}$ and to $100 \%$ at $48 \mathrm{~h}$ after inoculation (Table 1).

Digital image analysis of light micrographs. Settings in the digital image software that can be selected for generating in-focus images from a sequential series of micrographs are "patch size," which determines the size of equally focused regions that are used to generate the montage image, and "scan mode". The optimal settings in our research were found to be a patch size of 20 pixels for structures larger than $5 \mu \mathrm{m}$, and 5 to 10 pixels for structures of less than $5 \mu \mathrm{m}$, using the scan mode "blended depth". These patch sizes best accounted for the shape of appressoria and ILSs during line profiling. The blended depth mode was optimal to study the three-dimensional structure of the appressoria because depth maps and line profiles had a smooth appearance. Appressoria of $C$. acutatum had an asymmetrical, rounded shape with a diameter ranging between 4 and $6 \mu \mathrm{m}$ (Fig. 1). Digital analysis of montage images of appressoria with ILSs showed that the average diameter of the ILS was $0.7 \mu \mathrm{m}$ and that the ILS was surrounded by a dark ring structure of an average diameter of $1.4 \mu \mathrm{m}$ (Table 2).

A qualitative analysis of the depth relief in the ILS regions of appressoria was done by generating depth maps (i.e., depth relief images). The depth maps showed considerable differences in depth relief between appressoria without ILSs (Fig. 2B) and with ILSs (Fig. 2E and H). Appressoria without ILSs showed almost no depth relief, whereas appressoria with ILSs exhibited an extensive depth relief. The maximum depth in the appressorium corresponded to the ILS region based on the lowest in-focus image of the montage image.

The Auto-Montage software provides depth map values under any location of the image and depth reliefs were quantified after drawing line profiles for selected XY lines in the montage image
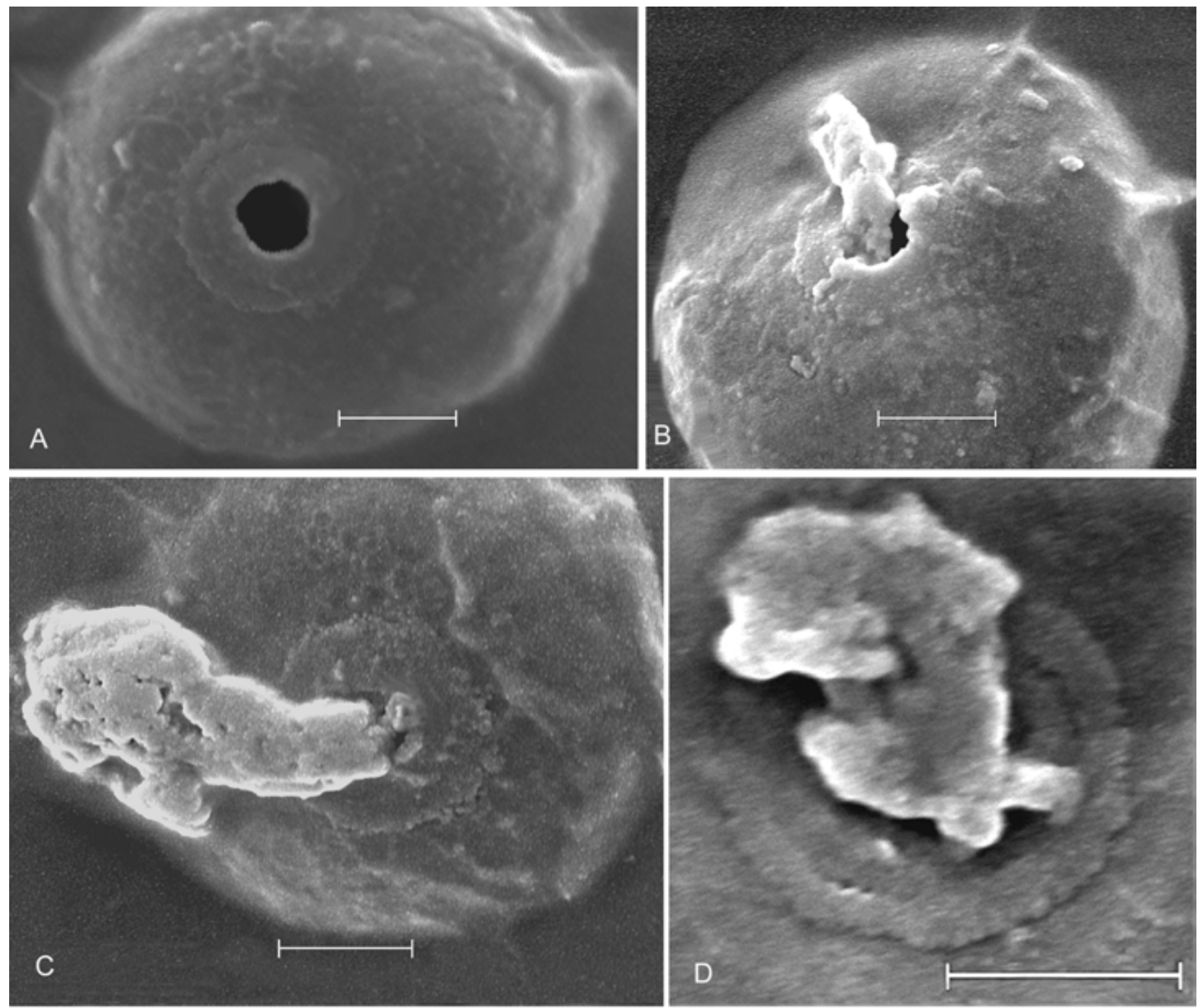

Fig. 5. Scanning electron micrographs of inverted, tape-mounted appressoria of Colletotrichum acutatum from almond leaves. Bars $=1 \mu \mathrm{m}$. A, Appressorium with penetration pore but with no peg. B to D, Appressoria with penetration pegs extruding through the penetration pores that are surrounded by a ring. The facing sides of the appressoria are flattened by host contact. The wall of the penetration peg is not continuous with the outer wall of the appressorium. B, Appressorium $12 \mathrm{~h}$ after inoculation with a penetration peg. C, Appressorium $24 \mathrm{~h}$ after inoculation with a penetration peg. D, Penetration peg $24 \mathrm{~h}$ after inoculation at higher magnification. 
(Fig. 2A, D, G, and J). Thus, line profile analyses of montage images allowed us to quantify the differences in depth within the appressoria. Line profiles of appressoria without ILSs showed very little depth relief and this relief originated from in-focus images of the appressorium that were $\approx 1$ to $3 \mu \mathrm{m}$ above the estimated leaf surface (Fig. 2C). In contrast, appressoria with ILSs had a pronounced depth relief in the ILS region that extended $\approx 0.1$ to $1.8 \mu \mathrm{m}$ below the leaf surface (Fig. $2 \mathrm{~F}$ and $\mathrm{G}$ ), indicating that the montage image information in the ILS region originated from images below the contact zone between appressorium and leaf surface.

Additional analyses of the ILS were done by studying shorter line profiles ( 1 to $3 \mu \mathrm{m}$ long) that were entirely located within the appressoria. This image analysis revealed that the ILS region has a convex shape (Fig. 2I). The upper in-focus planes of the ILS were located at the edge of the ILS and corresponded to a dark ring structure surrounding the ILS (Figs. 2G and H, 3A and B). In contrast, the lowest in-focus planes of the ILS were located within the ILS (Fig. 2H and I) and the estimated leaf surface was above the focal planes within the ILS region (Fig. 2I). Thus, the structure that was defined by the line profile of the ILS was protruding out of the dark ring that surrounded the ILS (Fig. 2I).

To calibrate the depth relief of the appressoria in relation to the leaf surface, we used carbon particles that were $\approx 1 \mu \mathrm{m}$ in diameter as surface markers (Fig. 3A, arrows). Depth maps (Fig. 2B) and line profiles (Fig. 2C) of images with carbon particles on the leaf surface confirmed that the depth relief in the ILS was below the leaf surface.

The depth relief within the ILS was quantified at different stages of appressorial development (i.e., times after inoculation). There was an increase in depth relief within the ILS at longer times after inoculation (Fig. 4). The regression model $Y=-0.09+$ $0.02 X$ was significant $(P<0.0001)$ and had an $R^{2}$ of 0.97 . The average depth relief was $\approx 0.2 \mu \mathrm{m}$ at $12 \mathrm{~h}$ after inoculation and $0.9 \mu \mathrm{m}$ at $48 \mathrm{~h}$ after inoculation. There was no increase in depth after longer incubation periods.

Digital image analysis of light micrographs and SEM of tape-mounted, inverted appressoria. Tape-mounted inverted

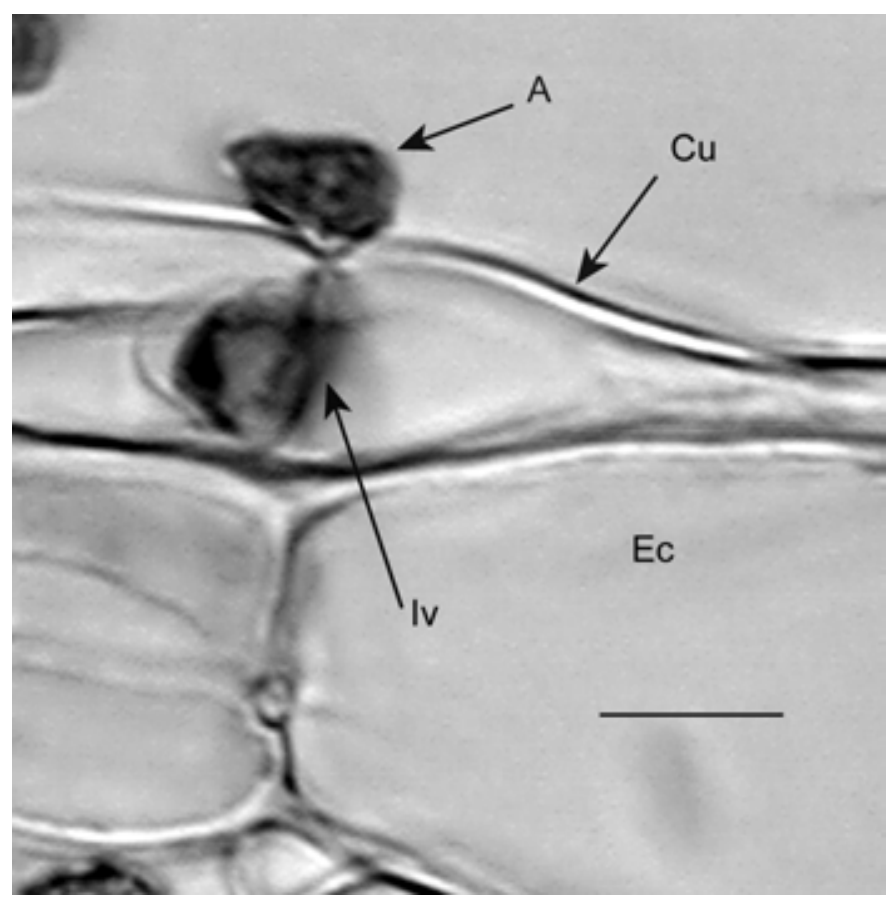

Fig. 6. Light micrograph of a cross section through an almond leaf $36 \mathrm{~h}$ after inoculation with conidia of Colletotrichum acutatum. The infection peg can be seen between the appressorium (A) and the subcuticular infection vesicle (Iv). $\mathrm{Cu}=$ cuticle, $\mathrm{Ec}=$ epidermal cell. $\mathrm{Bar}=5 \mu \mathrm{m}$. appressoria that were detached from the leaf tissue also were examined by digital image analysis (Fig. 2J). Depth maps and line profiles of the ILS region demonstrated that, for these inverted appressoria, the ILS was located in the upper focal planes (i.e., the area of the appressoria that was originally in contact with the leaf surface) (Fig. 2K and L). Scanning electron micrographs of these inverted appressoria are shown in Figure 5A to D. Appressoria were flattened at the base and were 4 to $6 \mu \mathrm{m}$ in diameter. A penetration pore was visible in the center of the appressorium that was surrounded by a ring. The position and the dimensions of these latter structures were very similar to those obtained in the digital image analyses of light micrographs of the ILS (Table 2). Approximately $50 \%$ of the detached appressoria that were sampled $12 \mathrm{~h}$ after inoculation showed a structure protruding from inside the penetration pore (Fig. $5 \mathrm{~B}$ to $\mathrm{D}$ ), whereas the remaining appressoria only showed a pore (Fig. 5A). The wall of the protruding structure was not continuous with the outer appressorial wall.

Histology of fungal penetration and colonization. Cross sections of leaves sampled 24 to $36 \mathrm{~h}$ after inoculation showed appressoria with penetration pegs and infection vesicles (Fig. 6). Pegs and infection vesicles were found below the cuticle. Hyphae were observed to grow within the epidermal wall and within epidermal cells before continued colonization of the leaf mesophyll tissue.

\section{DISCUSSION}

In this study, digital image analysis software was used to characterize the ILS in light micrographs of appressoria of C. acutatum on almond leaves. Appressoria with defined morphological appearances were selected for the analysis and our results provide the first direct evidence that the ILS corresponds to the appressorial pore and its developing infection peg. The ILS generally has been related to the penetration pore due to the absence of melanin (25) and because openings are present on the underside of appressoria that are detached from the leaf surface (3). Our work corroborates the current understanding that the ILS corresponds to the pore region (16-18). Our conclusions are based on several findings. First, in a time-sequence study, the ILS had an increasing depth relief between incubation times of 12 and $48 \mathrm{~h}$ after inoculation. Second, image analysis of the focal planes within the ILS showed that the in-focus images of the ILS originated from an area just below the leaf surface. In addition, line profile studies of inverted, tape-mounted appressoria with ILSs indicated a protruding structure in the ILS region. Conclusions from the digital image analysis of light micrographs were substantiated by histological and SEM studies. Thus, sectioning of inoculated almond leaves demonstrated that, $36 \mathrm{~h}$ after inoculation, the fungus had formed appressoria with penetration pegs and infection vesicles (Fig. 6). After this time period, hyphae grew subcuticularly and intracellularly (J. Diéguez-Uribeondo, unpublished data). In SEM studies, the presence of the protruding structure on inverted appressoria was confirmed. Dimensions of this structure and of a ring surrounding the pore were very similar using both SEM and image analysis. Therefore, this protruding structure is most likely the infection peg of the fungus. Some appressoria showed only a pore but no infection peg. This was most likely due to mechanical damage of the sample during processing for microscopy. Scanning electron micrographs also suggest that the wall of the penetration peg is not continuous with the outer appressorial wall, but arises from the inside of the pore. This observation is in agreement with ultrastructural studies on the penetration of other Colletotrichum spp. $(20,25,29)$ that show that the wall of the penetration peg is continuous with the new wall material that accumulates around the pore (i.e., the wall overlay or the appressorial cone). In addition, under our experimental conditions for the almond-C. $\mathrm{acu}$ tatum system, the penetration pore and peg were formed in a close 
sequence, and there was no quiescent phase between these two stages as has been described for Magnaporthe grisea (14). As in many other Colletotrichum spp.-host interactions (26), however, we consider that a quiescent phase does occur during infection of almond by $C$. acutatum. Infections as visualized by the presence of appressoria with ILSs may not develop into visual lesions if environmental conditions are not favorable (J. E. Adaskaveg and H. Förster, unpublished data). Therefore, infections may become quiescent after penetration pore and peg formation in the almondC. acutatum interaction similar to $C$. gloeosporioides on avocado and mango fruit (26).

In our time-sequence study, appressoria and ILSs developed in a similar sequence after inoculation, as described in a study with C. acutatum on strawberry (18). Thus, ILSs were first visible $12 \mathrm{~h}$ after inoculation and incubation at $20^{\circ} \mathrm{C}$. Using digital image analysis, early events in the penetration process as measured by increasing depth relief could be followed. Structural outlines as defined by different in-focus planes could be measured in fractions of a micron that is equivalent to the theoretical limit of resolution for light microscopy of $0.2 \mu \mathrm{m}$ (30). Depth relief did not increase after an incubation period of $48 \mathrm{~h}$. This can be explained by the limits in microscopic resolution in the deeper tissue areas. An increase in depth in the ILS has not been previously described by others. It is possible that, in other host-pathogen systems, infections are arrested at a very early stage, such as in an incompatible interaction, as was described for $C$. lagenarium in systemically protected cucumber leaves (31) or in latent infections as described for C. gloeosporioides (7). The application of digital image analysis in these systems could add more information on early penetration events.

Our results demonstrate that digital image analysis can add another dimension to the microscopic study of plant-pathogen interactions without the use of expensive and time-consuming techniques. In addition, multiple microscopic images to be analyzed can be specifically selected. Thus, a direct correlation between a particular structure and its three-dimensional shape can be made as with more advanced techniques, such as the use of fluorescent proteins and laser scanning confocal microscopy $(6,13)$. Using the new technology that we employed, the effect of environmental and host factors on initial stages in the infection process now can be easily quantified using a large sample size.

\section{ACKNOWLEDGMENTS}

We thank the Almond Board of California for financially supporting this project and A. Soto-Estrada for technical assistance in the histological studies.

\section{LITERATURE CITED}

1. Adaskaveg, J. E., Förster, H., Hartin, R. J., Connell, J. H., Teviotdale, B., and Duncan, R. 1998. Almond anthracnose in California-A new preand postharvest fungal disease outbreak. Pages 553-561 in: Proc. 2nd Int. Symp. Pistachios and Almonds. L. Ferguson and D. Kester, eds. Acta Hortic. 470. Int. Soc. Hortic. Sci. Leuven, Belgium.

2. Adaskaveg, J. E., and Hartin, R. J. 1997. Characterization of Colletotrichum acutatum isolates causing anthracnose of almond and peach in California. Phytopathology 87:979-987.

3. Bailey, J. A., O'Connell, R. J., Pring, R. J., and Nash, C. 1992. Infection strategies of Colletotrichum species. Pages 88-120 in: Colletotrichum: Biology, Pathology and Control. J. A. Bailey and M. J. Jeger, eds. CAB International, Wallingford, England.

4. Bartnicki-Garcia, S., Bracker, C. E., Gierz, G., Lopez-Franco, R. M., and Lu, H. 2000. Mapping the growth of fungal hyphae: Orthogonal cell wall expansion during tip growth and the role of turgor. Biophys. J. 79:23822390.

5. Brown, G. E. 1977. Ultrastructure of the penetration of ethylene-degreened Robison tangerines by Colletotrichum gloeosporioides. Phytopathology 67:315-320.

6. Czymmek, K. J., Bourett, T. M., Sweigard, J. A., Caroll, A., and
Howard, R. J. 2002. Utility of cytoplasmatic fluorescent proteins for live-cell imaging of Magnaporthe grisea in planta. Mycologia 94:280289.

7. Daykin, M. E., and Milholland, R. D. 1984. Histopathology of ripe rot caused by Colletotrichum gloeosporioides on muscadine grape. Phytopathology 74:1339-1341.

8. Förster H., and Adaskaveg, J. E. 1999. Identification of subpopulations of Colletotrichum acutatum and epidemiology of almond anthracnose in California. Phytopathology 89:1056-1065.

9. Glauert, A. M. 2000. Fixation, Dehydration, and Embedding of Biological Specimens. A. M. Glauert, ed. Elsevier North Holland Inc., New York.

10. Grove, S. N., Bracker, C. E., and Moore, D. J. 1970. An ultrastructural basis for hyphal tip growth in Pythium ultimum. Am. J. Bot. 57:245-266.

11. Hoch, H. C. 1977. Use of permanganate to increase electron opacity of fungal walls. Mycologia 6:1209-1213.

12. Hoch, H. C. 1991. Preservation of cell ultrastructure by freeze-substitution. Pages 1-16 in: Electron Microscopy of Plant Pathogens. K. Mendgen and D. E. Lesemann, eds. Springer Verlag, Berlin.

13. Horowitz, S., Freeman, S., and Sharon, A. 2002. Use of green fluorescent protein-transgenic strains to study pathogenic and nonpathogenic lifestyles in Colletotrichum acutatum. Phytopathology 92:743-749.

14. Howard, R. J., and Valent, B. 1996. Breaking and entering: Host penetration by the fungal rice blast pathogen Magnaporthe grisea. Annu. Rev. Microbiol. 50:491-512.

15. Landes, M., and Hoffmann, G. M. 1979. Zum Keimungs- und Infektionsverlauf bei Colletotrichum lindemuthianum auf Phaseolus vulgaris. Phytopathol. Z. 95:259-273.

16. Latunde-Dada, A. O., O'Connell, R. J., Nash, C., and Lucas, J. A. 1999. Stomatal penetration of cowpea (Vigna ungiculata) leaves by Colletotrichum acutatum causing latent anthracnose. Plant Pathol. 48:777785.

17. Latunde-Dada, A. O., O’Connell, R. J., Nash, C., Pring, R. J., Lucas, J. A., and Bailey, J. A. 1996. Infection process and identity of the hemibiotrophic anthracnose fungus (Colletotrichum destructivum) from cowpea (Vigna ungiculata). Mycol. Res. 100:1133-1141.

18. Leandro, L. F. S., Gleason, M. L., Nutter, F. W., Jr., Wegulo, S. N., and Dixon, P. M. 2001. Germination and sporulation of Colletotrichum acutatum on symptomless strawberry leaves. Phytopathology 91:659664.

19. Mendgen, K., and Deising, H. 1993. Infection structures of fungal plant pathogens-A cytological and physiological evaluation. New Phytol. 124:193-213.

20. O'Connell, R. J., and Bailey, J. A. 1991. Hemibiotrophy in Colletotrichum lindemuthianum. Pages 211-222 in: Electron Microscopy of Plant Pathogens. K. Mendgen and D. E. Lesemann, eds. Springer-Verlag, Berlin.

21. O'Connell, R. J., Perfect, S. E., Hughes, H. B., Carazaniga, R., and Bailey, J. A. 2000. Dissecting the cell biology of Colletotrichum infection processes. Pages 57-76 in: Colletotrichum: Host Specificity, Pathology, and Host-Pathogen Interaction. D. Prusky, S. Freeman, and M. B. Dickman, eds. The American Phytopathological Society, St. Paul, MN.

22. Pain, N. A., O'Connell, R. J., and Green, J. R. 1995. A plasma membrane-associated protein is a marker for differentiation and polarization of Colletotrichum lindemuthianum appressoria. Protoplasma 188:1-11.

23. Parbery, D. G., and Blakeman, J. P. 1978. Effect of substances associated with leaf surfaces on appressorium formation by Colletotrichum acutatum. Trans. Br. Mycol. Soc. 70:7-19.

24. Perfect, S. E., Hughes, H. B., O’Connell, R. J., and Green, J. 1999. Colletotrichum: A model genus for studies on pathology and fungal-plant interactions. Fungal Genet. Biol. 27:186-198.

25. Politis, D. J., and Wheeler, H. 1973. Ultrastructural study of the penetration of maize leaves by Colletotrichum graminicola. Physiol. Plant Pathol. 3:465-471.

26. Prusky, D., and Plumbley, R. A. 1992. Quiescent infections of Colletotrichum in tropical and subtropical fruits. Pages 289-307 in: Colletotrichum: Biology, Pathology and Control. J. A. Bailey and M. J. Jeger, eds. CAB International, Wallingford, England.

27. Timmer, L. W., and Brown, G. E. 2000. Biology and control of anthracnose diseases of citrus. Pages 300-316 in: Colletotrichum: Host Specificity, Pathology, and Host-Pathogen Interaction. D. Prusky, S. Freeman, and M. B. Dickman, eds. The American Phytopathological Society, St. Paul, MN.

28. Waller, J. M. 1992. Colletotrichum diseases of perennial and other cash crops. Pages 167-185 in: Colletotrichum: Biology, Pathology and Control. J. A. Bailey and M. J. Jeger, eds. CAB International, Wallingford, England. 
29. Wharton, P. S., Julian, A. M., and O'Connell, R. J. 2001. Ultrastructure of the infection of Sorghum bicolor by Colletotrichum sublineolum. Phytopathology 91:149-172.

30. Wischnitzer, S. 1962. Introduction to Electron Microscopy. Pergamon Press, New York.

31. Xuei, X. L., Järlfors, U., and Kuc, J. 1988. Ultrastructural changes associated with induced systemic resistance of cucumber to disease: host response and development of Colletotrichum lagenarium in systemically protected leaves. Can. J. Bot. 66:1028-1038.

32. Zulfiqar, M., Brlansky, R. H., and Timmer, L. W. 1996. Infection of flower and vegetative tissues of citrus by Colletotrichum acutatum and C. gloeosporioides. Mycologia 88:121-128. 